\title{
Escepticismo y suspensión del juicio en la teoría nominalista del conocimiento de Francisco Suárez
}

\author{
Oscar YANGaLI \\ Pontificia Universidad Católica del Perú
}

Resumen: El objetivo del presente trabajo es ofrecer una reflexión acerca de la teoría del conocimiento de Francisco Suárez y su nominalismo a la luz del pensamiento escéptico de los Esbozos Pirrónicos de Sexto Empírico. Es Sexto quien nos permite comparar la epistemología de Suárez con un sistema dinámico del conocimiento. Para esto, en primer lugar, examino el escepticismo de Sexto y la ontología nominalista de Suárez. En segundo lugar, analizo la representación mental concebida por Sexto análogamente a los conceptos formal y objetivo de Suárez, procediendo también a un análisis en torno al concepto de relación en ambos autores. Finalmente, procedo a desarrollar un examen acerca del concepto de creencia en cuanto son patentes y reconocidas ciertas restricciones en la configuración de nuestros juicios.

Palabras clave: Sexto, Suárez, conocimiento, relación, creencia.

Abstract: The aim of this study is to offer a reflection on the theory of knowledge of Francisco Suárez and his nominalism in the light of Sextus Empiricus' sceptical thought in his Outlines of Pyrrhonism. It is Sextus who allows us to compare the epistemology of Suárez with a dynamic system of knowledge. With this in view, in the first place, I examine Sextus's scepticism and Suárez's nominalist ontology. In the second place, I analyse the mental representation as conceived by Sextus in analogy with the formal and objective concepts of Suárez, and I also discuss the concept of relation in both authors. Finally, I proceed to examine the concept of belief inasmuch as certain restrictions in the configuration of our judgements are manifest and recognized.

Keywords: Sextus, Suárez, knowledge, relation, belief. 


\section{§ 1. Introducción}

¿Qué podría aportar el pensamiento de Sexto Empírico a la teoría del conocimiento de Francisco Suárez? ¿Es posible desentrañar algún paralelo respecto de algunas de sus teorías acerca de nuestras creencias? Para la presente investigación hay que exponer el pensamiento de Sexto Empírico en torno a nuestra posibilidad de conocer ya que, como veremos a continuación, el pensamiento de Sexto nos exhorta a examinar cómo es que se forman nuestras creencias. A su vez, hay que exponer los fundamentos en los que se apoya Francisco Suárez para desarrollar una teoría nominalista del conocimiento. $Y$ es que al estar ambos pensamientos inclinados hacia la experiencia de lo sensible y lo singular como inicio de todo conocimiento, podemos encontrar semejanzas en los postulados respecto de su configuración. La importancia de este trabajo radica en advertir que las posturas de ambos autores pueden ser vinculadas a través del examen del conocimiento.

\section{$\S 2$. Escepticismo y nominalismo}

Sostengo que ambas posturas pueden ser compatibles, en primer lugar, sobre la base del común punto de partida de estos autores, a saber, la percepción del fenómeno por medio de las sensaciones según Sexto y la experiencia que tenemos del singular de acuerdo a Suárez. Por este motivo, si bien puede existir distancia entre ambos autores respecto de la época, el lugar o diversidad de temas, trataré de aprovechar las afinidades entre ambas posturas con la finalidad de analizar una similitud en favor del objetivo del trabajo.

Conocido por pertenecer a una línea escéptica pirrónica, Sexto nos ofrece su parecer acerca de cómo se presentan los fenómenos a nuestra percepción y porqué debemos mantenernos firmes en torno a lo sensible. En los Esbozos Pirrónicos' Sexto expone lo que entiende por escepticismo con las siguientes palabras: "Y el escepticismo es la capacidad de establecer antítesis en los fenómenos y en las consideraciones teóricas, según cualquiera de los

I Para el presente trabajo utilizo la siguiente edición: Empírico, Sexto, Esbozos Pirrónicos, introducción, traducción y notas de Antonio Gallego Caoy Teresa Muñoz Diego, Madrid: Gredos, 1993. Citada en adelante como EP. 
tropos; gracias a la cual nos encaminamos -en virtud de la equivalencia entre las cosas y proposiciones contrapuestas- primero hacia la suspensión del juicio y después hacia la ataraxia. / Hablamos de "capacidad», desde luego no por capricho sino sencillamente en el sentido de que uno sea capaz. / Aquí entendemos por «fenómenos» lo sensible; por lo que definimos lo «teórico» por oposición a ellos" ${ }^{2}$.

Según Sexto, el escepticismo es comprendido en cuanto somos capaces de establecer antítesis a los fenómenos y también a las consideraciones teóricas según los tropos ${ }^{3}$. Es por esta capacidad que nosotros podemos realizar la suspensión del juicio en cuanto respondemos con una antítesis o proposición contrapuesta equivalente a la enunciada en primera instancia. Así, la suspensión del juicio consistirá precisamente en establecer antítesis equivalentes o proposiciones contrapuestas con la finalidad de llegar a la ataraxia ${ }^{4}$. $Y$ es que tenemos dicha capacidad según Sexto. Además, refiere Sexto a lo que es sensible como fenómeno mientras que a la especulación la refiere como consideración teórica y lógicamente opuesta a lo que es sensible.

El escepticismo de Sexto no será sino una actitud crítica respecto de las opiniones dadas acerca de los fenómenos o las consideraciones teóricas. Y es que, según Sexto, cualquier afirmación acerca de las cosas es susceptible de ser amonestada por otra afirmación contraria equivalente. No podemos tener por absolutamente cierta a ninguna afirmación por el solo hecho de que siempre podremos contraponerle otra que la niegue o que por lo menos la haga no ser del todo evidente. Sin embargo, como sabemos que no podemos otorgarle a todas las creencias el mismo valor de verdad, entonces nos encontramos en una turbación espiritual que obviamente no nos deja en paz.

$2 \quad E P, \mathrm{I}, 8-9$, pp. 53-54.

3 En lo sucesivo no me detendré en mencionar ni desarrollar los diez tropos que considera Sexto para ser capaces de establecer estas antítesis, pues me concentraré solo en el octavo tropo acerca de la relación en el segundo capítulo del presente trabajo -por razones de especificidad en la similitud con el pensamiento de Suárez.

$4 \quad$ Llamada también serenidad de espíritu (cf. EP, I, I0, p. 55), tema el cual no desarrollaré tampoco en el presente trabajo por estar lejos del alcance del mismo. Aunque, cabe especificar de todas maneras que Sexto la comprende con las siguientes palabras: "Pues bien, desde ahora decimos que el fin del escepticismo es la serenidad de espíritu en las cosas que dependen de la opinión de uno y el control del sufrimiento en las que se padecen por necesidad" (EP, I, 25, p. 6I). 
Así, es prudente suspender el juicio como una reacción ante aquella situación que no nos satisfice. Por ello, Bailey indica que: "Naturally the extensive غ̇ं that results from these developments starts out as little more than a desperate expedient that offers the future Pyrrhonist a temporary escape from an otherwise intolerable situation" ${ }^{5}$. Podemos decir, entonces, que la suspensión del juicio nos sirve como aquella actitud ante la ausencia de respuesta que se buscó en primera instancia con la finalidad de evitar caer en dogmatismos. "Que el escéptico no dogmatiza no lo decimos en el sentido de dogma en que algunos dicen que «dogma es aprobar algo en términos más o menos generales», pues el escéptico asiente a las sensaciones que se imponen a su imaginación; por ejemplo, al sentir calor o frío, no diría «creo que no siento calor» o «no siento frío». Sino que decimos que no dogmatiza en el sentido en que otros dicen que «dogma es la aceptación en ciertas cuestiones, después de analizadas científicamente, de cosas no manifiestas»; el pirrónico en efecto no asiente a ninguna de las cosas no manifiestas".

El escéptico busca evitar a toda costa dogmatizar, es decir, evitar sostener afirmaciones inamovibles acerca de las cosas. Aunque cabe decir que el escepticismo de Sexto asiente a las sensaciones que se nos imponen, lo que le interesa sobre todo a Sexto es evitar el dogmatismo en cuanto a creencias fijas sobre la realidad precisamente por no ser las creencias cosas manifiestas como lo son las sensaciones.

El escéptico pirrónico de Sexto no pretende dogmatizar pues siempre una creencia puede ser contradicha por otra equivalente. De esa manera también en primera instancia no es posible detenernos en las razones de una creencia como si fueran realmente mejores. Así, Brochard señala: “(...) le parece que contra los dogmáticos todos los medios son buenos, tanto más cuanto que en su calidad de escéptico no tiene que elegir entre las buenas razones y las malas: no debe saber, no sabe, si hay entre ellas una diferencia"7. Para Sexto no es posible determinar que una creencia sea mejor que otra de manera conclusiva, sino que lo que podemos hacer es contraponer una opinión

5 Bailey, Alan, Sextus Empiricus and Pyrrhonean Scepticism, Oxford: Oxford University Press, 2002, p. I2I.

$6 \quad E P, \mathrm{I}, \mathrm{I3}, \mathrm{p} .56$.

7 Brochard, Víctor, Los escépticos griegos, traducción de Vicente Quinteros, Buenos Aires: Editorial Losada, 1945, p. 392. 
equivalente a otra opinión con el propósito de suspender el juicio. Esta es la tesis de Sexto, aunque es importante no olvidar que el escepticismo pirrónico de Sexto no consiste en una desconfianza absoluta por la posibilidad de conocer sino, como ya se señaló, en la capacidad de establecer proposiciones contrapuestas o de establecer antítesis a las mencionadas en una primera instancia con la finalidad de suspender el juicio.

Ahora, paso a exponer la teoría nominalista del conocimiento que defiende Francisco Suárez para iniciar una búsqueda de similitud con Sexto en este primer punto; se trata, como mencioné, de la similitud que tiene la experiencia del singular en Suárez respecto de la percepción del fenómeno en Sexto. En las Disputaciones metafísicas ${ }^{8}$ podemos leer lo siguiente: "Hay que suponer que el ente individual o singular se opone al ente común o universal, no sólo relativamente, en cuanto que según la comparación de la mente o la consideración dialéctica el individuo está sometido a la especie, puesto que eso ni conviene a toda naturaleza individual (...); se opone, por consiguiente, de modo cuasi privativo, casi del mismo modo como se opone la unidad a la multitud. En efecto, se dice común o universal aquello que según una razón única se comunica a muchos, o se encuentra en muchos; $y$, en cambio, se llama numéricamente uno, o singular e individual aquello que de tal forma es un ente que según aquella razón de ente por la que se dice uno, no es comunicable a muchos como inferiores y subordinados a él (...)"’.

Para Suárez todo ente individual se encuentra relativamente opuesto a todo ente universal, pues precisamente un ente individual no es universal, y también de modo privativo en cuanto no lo refiere sino al contrario. Según Suárez todo lo que existe como ente es un ente singular o individual. Y es que la razón universal de las cosas solo se dice por una razón de comunicación hacia los inferiores, como el hombre en cuanto tal que puede dividirse en los hombres concretos.

$8 \quad$ Utilizo en el presente trabajo la edición: Suárez, Francisco, Disputaciones metafísicas, edición y traducción de Sergio Rábade Romeo, Salvador Caballero Sánchez y Antonio Puigcerver Zanón, Madrid: Biblioteca Hispánica de Filosofía, Editorial Gredos, 1960. Citada en adelante como $D M$.

I 229 DM, V, Sec. I, p. 564. 
Así, cabe decir que la razón individual no permite dicha razón de comunicación hacia inferiores, razón por la cual un hombre es singular o unidad indivisible, pues de su división no surgirían varios hombres concretos. Entonces, debemos decir que todas las cosas que son entes actuales o que existen, son singulares e individuales. "Explicada, por tanto, así la razón de individuo o de ente singular, hay que decir que todas las cosas que son entes actuales o que existen 0 pueden existir inmediatamente, son singulares e individuales" ${ }^{\circ}$. Y es que ser singular consiste en ser una entidad que no se diferencia de sí misma aunque se diferencia de las demás. Pereira dice: "Singularity is opposed to plurality: to be at once singular (undifferentiated from itself) and plural (differentiated into many distinct entities which are the same as itself) is a contradiction"l".

Por esta razón, Suárez hará énfasis en el ente singular como lo único real existente, donde la individualidad pasa a ser la naturaleza propia de cada ente: "Por lo dicho hasta ahora en contra de las opiniones anteriores, parece que resta, como tras una enumeración suficiente de las partes, que toda sustancia singular [por sí misma o por su entidad es singular] y que no necesita ningún otro principio de individuación fuera de su entidad, o fuera de los principios intrínsecos de que consta su entidad. Pues si tal sustancia físicamente considerada es simple, por sí misma y por su simple entidad es individual; (...) Por lo cual, como la unidad individual en lo que tiene de formal no puede añadir nada positivo real sobre la entidad individual, ya que en este punto subsiste la misma razón acerca de ella y de toda unidad, así el fundamento positivo de esta unidad en cuanto a la negación que dice, no puede añadir nada positivo, hablando físicamente, a aquella entidad, que se denomina una e individual; luego aquella entidad, por sí misma, es el fundamento de esta negación y en este sentido se afirma en aquella opinión que por sí misma es el principio de individuación. Porque no niega esta opinión que en aquella entidad individual pueda distinguirse con la razón la naturaleza común de la entidad singular, y que de este modo el individuo añada sobre la especie algo conceptualmente distinto, lo cual, según la consideración metafísica, tiene razón de diferencia individual [differentiae individualis], tal como se ha dicho en la sección precedente; (...)"'2.

$10 \quad$ Ibid., p. 566.

II Pereira, José, Suárez: Between Scholasticism \& Modernity, Milwaukee: Marquette University Press, 2007, p. 70.

12 DM, V, Sec. 6, pp. 644-645. 
Según Suárez, es el individuo o ente singular el que añade a la naturaleza común algo real por razón de lo cual es tal individuo y le conviene aquella negación de divisibilidad en varios semejantes. Es decir, el ente singular o individual es el que es real y no la naturaleza común que no es sino una semejanza entre los singulares o individuales. Solo el individuo es real, no una naturaleza común por la que un individuo es semejante a otros individuos. Es el concepto de differentia individualis el que le permite a Suárez rechazar la entidad de la naturaleza común o universal. La noción de differentia individualis es lo que nos permite comprender qué se adiciona a la naturaleza común para contraer la especie y constituir al individuo. Así, la entidad individual por sí misma es la que fundamenta la contracción de la especie para constituirse un ente individual. Ahora, esta contracción consiste en una adición, pero solo conceptual o de razón aplicada a la naturaleza común. Esta contracción o principio de individuación es, por tanto, la diferencia individual y es suficiente para constituir al ente singular.

Es así que el pensamiento de Suárez se encuentra cimentado sobre la base e influencia de una ontología nominalista en tanto que se rechaza la realidad de la naturaleza común o universal en favor del individuo o singular' ${ }^{13}$. Señala Secada: "The individuality of Suárez's nominalist thesis is the individuality of things which are indivisible and incommunicable according to their nature or to that account which captures what they are in themselves"14. Por ello, la teoría del conocimiento de Suárez responderá a este nominalismo en cuanto que las definiciones, si bien están referidas a nociones universales, encuentran su base o principio en las cosas singulares y no respecto de universales absolutos. Ya luego por medio de las semejanzas es que se elaborarán éstos.

En suma, tanto Sexto como Suárez nos remiten a unas posiciones que se constituyen a partir de los fenómenos en cuanto cosas manifiestas o de la

I3 Doy por supuesto el desarrollo que tiene Suárez acerca de la forma individual. Y es que para Suárez el principio mismo de individuación no se remite ni a la materia ni a una forma general, sino a la forma del individuo mismo. Suárez comienza un apartado de la sección correspondiente afirmando por este motivo: “(...) hay que decir que la forma sustancial es ésta intrínsecamente por su misma entidad, de la cual se toma según el último grado o realidad su diferencia individual" (ibid., p. 648).

14 Secada, Jorge, "Suárez's nominalist master argument: metaphysical disputations 5, I", en: Suárez's Metaphysics in its Historical and Systematic Context, Berlín/Boston: Walter de Gruyter, 2014, p. 224. 
experiencia de las cosas en cuanto singulares. Esto nos permite hallar una primera similitud en ambos autores, pues encontramos una fundada desconfianza o desaprobación hacia las definiciones basadas en creencias que responden a consideraciones teóricas o universales como cimiento. Por tanto, vemos que el escepticismo de Sexto y el nominalismo inmerso en Suárez son posturas compatibles desde este examen.

\section{$\S 3$. El conocimiento}

En este segundo capítulo hay que explicar más acerca de cómo es que se procede en la investigación por el conocimiento, tanto según Sexto como según Suárez. Es en este espacio de la investigación donde ambos autores expresan sus maneras de evaluar cómo es que pretendemos conocer las cosas. Es necesario examinar este punto en el pensamiento tanto de Sexto como de Suárez, pues creemos que todo conocimiento se obtiene a partir de un ejercicio de nuestra inteligencia por medio de las representaciones 0 conceptos mediante los cuales establecemos relaciones ya entre nosotros y el mundo o entre las cosas.

No podemos escapar al intento de aproximarnos al mundo, de manera que Sexto también siente la necesidad de expresarse acerca de qué debe hacer el escéptico respecto de los fenómenos. Sexto nos indica con las siguientes palabras el criterio de orientación escéptica: "Pues bien, decimos que el criterio de la orientación escéptica es el fenómeno, llamando implícitamente así a la representación mental. Consistiendo, en efecto, en una impresión y en una sensación involuntaria, es incuestionable; por lo cual, nadie seguramente disputará sobre si el objeto se percibe en tal o cual forma, sino que se discute sobre si es tal cual se le percibe. / Atendiendo, pues, a los fenómenos, vivimos sin dogmatismos, en la observancia de las exigencias vitales, ya que no podemos estar completamente inactivos" ${ }^{\prime 5}$. Sexto nos señala que el escéptico debe reconocer a toda representación mental como fenómeno la cual es una impresión de las sensaciones. No solemos discutir sobre dicha percepción, sino sobre si se le percibe de una manera determinada para todos, pues es

$15 E P, \mathrm{I}, 22-23$, p. 60. 
ahí donde surgen las discrepancias. Sin embargo, debemos vivir conforme a la percepción de los fenómenos. Esto es, sin dogmatismo alguno, aunque será inevitable que nos encontremos inactivos ante los objetos como consecuencia de una exigencia vital.

De acuerdo a las palabras de Sexto podemos decir que la actividad que realizamos por conocer es también connatural a nosotros. Sin embargo, la posición que tiene Sexto respecto de esta necesidad es que debemos evitar a toda costa caer en algún dogmatismo, en cuanto terminemos en afirmaciones sobre las cosas que pretenden fijarse de manera incuestionable. Dice Pellegrin: “'External objects' here include what we call by this name, but also concepts that we form, and judgements that we make about these objects and concepts. Hence one falls into dogmatism as soon as one gives assent to an opinion"16. Por este motivo es importante para Sexto evitar a toda costa aceptar irreflexivamente cualquier opinión, porque nos comprometerá con juicios fijos e inamovibles, y ello nos volverá dogmáticos.

La teoría del conocimiento de Suárez posee también una explicación de cómo los conceptos se forman en nuestra mente. Ciertamente, solo por medio de la elaboración de los conceptos es que creemos conocer algo. Se sabe que Suárez hereda el problema escolástico acerca de los universales, los cuales debían ser explicados básicamente como si fueran reales o no. Para abordar este tema me concentraré en la explicación que tiene Suárez acerca de cómo es que las cosas singulares o individuales conforman los conceptos a partir de un acto de nuestro entendimiento.

Es necesario, entonces, mencionar las nociones de concepto formal y objetivo que Suárez maneja: "Se llama concepto formal al acto mismo o, lo que es igual, al verbo con que el entendimiento concibe una cosa o una razón común. Se le da el nombre de concepto, porque viene a ser como una concepción de nuestra mente; y se le llama formal, bien porque es la última forma de la mente, bien porque representa formalmente al entendimiento la cosa conocida, bien porque, en realidad, es el término formal e intrínseco de la concepción mental, consistiendo, por decirlo así, en esto su diferencia del concepto objetivo.

16 Pellegrin, Pierre, "Sextus Empiricus", en: Bett, Richard (ed.), The Cambridge Companion to Ancient Scepticism, Cambridge: Cambrigde University Press, 2010, p. 125. 
Llamamos concepto objetivo a la cosa o razón que, propia e inmediatamente, se conoce o representa por medio del concepto formal; por ejemplo: cuando concebimos un hombre, el acto que realizamos para concebirlo en la mente se llama concepto formal, en cambio, el hombre conocido y representado en dicho acto se llama concepto objetivo"17.

El concepto formal para Suárez consiste en la concepción o acto de concebir formalmente algo. Se llama concepto formal puesto que es una operación de la mente la cual formaliza lo que pretende conocer. Por otra parte, el concepto objetivo es la representación que se refleja del concepto formal. Y es que para Suárez el concepto objetivo no es sino la concepción que realiza nuestra mente por medio del concepto formal.

El concepto formal y el concepto objetivo conforman parte central en la teoría del conocimiento de Suárez. Esto porque es gracias a estos dos conceptos que se construye o elaboran nuestros conocimientos acerca de las nociones universales. Y es que según Suárez nosotros construimos el universal a partir de las semejanzas que se encuentran en los entes singulares ${ }^{18}$. No hay tal cosa como un universal subsistente en las cosas, el cual el entendimiento abstraiga. Según Suárez nosotros elaboramos nuestras definiciones o universales a partir de la semejanza que encontramos entre un conjunto de entes individuales. Por eso Suárez señala: “(...) la unidad del concepto objetivo no consiste en una unidad real y numérica, sino en una unidad formal o fundamental, que no es otra cosa que la conveniencia o semejanza antes propuesta"|9.

Ahora bien, podemos agregar a estas observaciones sobre Sexto y Suárez en torno a la representación y los conceptos las explicaciones que también nos brindan acerca de la relación. Esto es útil para el presente trabajo, pues podemos ver que en ambos autores la noción de relación juega un papel

17 DM, II, Sec. I, p. 36I.

I8 No me detengo en explicar la concepción base de los universales en Suárez por motivos de espacio y por ello la presupongo. En todo caso, bastará indicar que hay que saber que para Suárez el universal no se obtiene sino por una actividad realizada por nuestra mente, nunca anteriormente. El universal, por tanto, no es real ni se da en las cosas, sino que se da como un concepto unitario a partir de las semejanzas que encontramos en las cosas porque en la realidad no hay nada verdaderamente uno y realmente indiviso a excepción de la forma individual. Así, no hay unidad real universal sino solo a partir de semejanzas (cf. DM, VI, Sec. II, pp. 7I9-720).

19 DM, II, Sec. I, p. 384. 
fundamental, ya sea acerca de la percepción o de la asociación que hacemos entre las cosas.

Es aquí donde me detengo en mencionar el octavo tropo indicado por Sexto en el que se pronuncia acerca de la relación. Para Sexto la relación indica en principio que las percepciones de los objetos tienen siempre que ver con relación a alguien que percibe las cosas como le aparecen. Nuestra forma de percibir no puede ocurrir de otro modo: "El octavo tropo es el de «a partir del con relación a algo»; según el cual razonamos que, como todas las cosas son con relación a algo, mantendremos en suspenso el juicio sobre el qué son absoluta y objetivamente. Pero es preciso darse cuenta de una cosa: de que aquí, como en otras partes, usamos el «son» en lugar del «aparecen»; como diciendo implícitamente «todas las cosas aparecen como con relación a algo»”20.

Vemos que para Sexto las cosas siempre se dan con relación a algo. De modo que no es posible percibir algo absolutamente sino con relación a la percepción de dicho individuo. Por eso las cosas son siempre con relación a algo, donde son y aparecen los utilizamos como equivalentes. Así, la relación se funda siempre en la percepción de alguien ${ }^{21}$. Añade Sexto: "Pero también se puede razonar de un modo concreto el que todas las cosas son con relación a algo; de esta forma: las cosas diferenciadas ise distinguen o no de las cosas que no son con relación a algo? Si no se distinguen, ya son con relación a algo. Y si se distinguen, las cosas diferenciadas son con relación a algo, porque lo diferenciado se da con relación a algo, pues es pensado en relación con aquello de lo que se distingue"22.

Sexto indica que incluso al momento de aproximarnos hacia las cosas podemos cotejar que unas no se distinguen de otras así como también unas sí se distinguen de otras. Es decir, puede ocurrir que a nuestra percepción una cosa no se distinga de otra y que también veamos que una cosa sí se distingue de otra. En el primer caso, si unas cosas no se distinguen de otras entonces de

$20 \quad E P, \mathrm{I}, \mathrm{I} 35$, p. 94.

21 Es necesario reconocer para Sexto que quien juzga siempre lo hace en relación a su percepción, ya que las cosas aparecen según la percepción de alguien. Por eso, por ejemplo, menciona Sexto que la derecha aparece respecto de la izquierda. Todo se manifiesta según el animal, el hombre, el sentido o circunstancia (cf. EP, I, I35-I36, p. 94). 
todas maneras son con relación a algo, pues aparecen con relación a mi percepción; en el segundo caso, si unas cosas sí se distinguen de otras entonces también son con relación a algo, pues también se distinguen por quien las piensa distintas. Entonces, podemos decir que es imposible esquivar la relación en nuestra percepción. Woodruff resume al referirse al octavo tropo: "All things are relative because, for example, all things belong to species and genera, and these are relative to each other. Therefore we cannot state the nature of external objects with precision" ${ }^{23}$. Y es que, ya que todo refiere relación con algo, no es posible dar con alguna naturaleza real de las cosas.

Por otra parte, Suárez también nos ofrece una manera de entender la relación. Aunque la relación en Suárez no se dirige directamente hacia la percepción de las cosas, sí la sugiere indirectamente, pues solo lo que entiende Suárez por relación nos permite dilucidar precisamente la relación entre nuestros conceptos y los singulares. Menciona Suárez: "Hay que entender, por el contrario, que la relación expresa ciertamente alguna forma real y que denomina de manera intrínseca al propio relativo que constituye; pero, por su parte, no es ninguna realidad o modo distinto ex natura rei de toda forma absoluta, sino que en la realidad es una forma absoluta, mas no considerada absolutamente, sino en cuanto se refiere a otra que está incluida o connotada en la denominación relativa; de modo que la semejanza, por ejemplo, es alguna forma real existente en la cosa que se denomina semejante, pero ella no es realmente distinta de la blancura en cuanto a aquello que pone en la cosa que se llama semejante, sino sólo en cuanto al término que connota; así, la semejanza, en la cosa, no es más que la misma blancura en cuanto se refiere a otra blancura como poseedora de una razón idéntica o semejante" 24 .

La relación para Suárez no es algo real distinto de la cosa, sino que se denota por medio de la semejanza entre dicha cosa y la forma conceptual. En otras palabras, la relación entre el concepto y la cosa singular se da por medio de la semejanza. Así, esta relación encontrará su fundamento en la semejanza que guarda dicho singular respecto de otros que también podrán ser representados por la misma forma conceptual. De esta manera, por ejemplo, la semejanza

23 Woodruff, Paul, “The Pyrrhonian Modes”, en: Bett, Richard (ed.), The Cambridge Companion to Ancient Scepticism, p. 215.

24 DM, XLVII, Sec. II, p. 66I. 
de blancura que se encuentra en la cosa singular es la que permite referirme a la razón de blancura en general.

Por ese motivo Suárez también añade: "Y de ahí no se sigue que la relación de semejanza no sea nada, sino que se sigue únicamente que no es algo distinto de la blancura que antes existía en tal cosa blanca" ${ }^{25}$, ya que la blancura como universal no es algo real ni independiente de las cosas singulares blancas por semejanza. Y es que para Suárez no es posible llegar a una elaboración conceptual o universal prescindiendo de tales cosas singulares: “(...) cuando se dice que la denominación respectiva surge de la coexistencia de varias cosas absolutas sin ninguna adición real, (...) hay que entender que esa denominación requiere ciertamente la asociación o coexistencia de tales cosas o formas" 26 . Luego, podemos señalar que la relación es, según Suárez, la que nos permite concluir por qué razón no es posible tener conceptos universales absolutos en tanto reales e independientes de las cosas singulares. Si no se dan estos universales con relación a nuestra experiencia de los entes individuales, entonces no podemos elaborar universal alguno con nuestra mente.

Llegado a este punto, recapitulo las similitudes que encuentro entre el pensamiento de Sexto y el de Suárez respecto de cómo entienden nuestras representaciones y conceptos mentales. En el primer punto, vimos que tanto para Sexto como para Suárez solo es posible realizar investigación en la realidad por medio de la referencia a los fenómenos o entes singulares. Aquí he expuesto que si bien la relación para Sexto no refiere precisamente lo mismo que para Suárez, sin embargo, podemos inferir que en ambos autores el concepto de relación conduce a una conclusión similar en tanto que ambos pretenden señalar que cualquier representación o concepto mental en última instancia no se corresponde con alguna naturaleza común de las cosas en general si no es gracias a la percepción o la mente. Esto ya sea porque toda representación se da con respecto a alguien, o ya sea porque todo concepto se forja a partir de la confusión entre varios singulares o individuales -siendo, por tanto, el universal algo confuso y no preciso. Así, encontramos nuevamente una similitud entre Sexto y Suárez en torno a sus observaciones acerca de la elaboración de nuestros contenidos mentales y sus relaciones.

$\begin{array}{lll}130 & 25 & \text { Ibid., p. } 662 . \\ & 26 & \text { Ibid., p. } 664 .\end{array}$




\section{§ 4. La configuración y el dinamismo de la creencia}

Finalmente, en este tercer capítulo paso a examinar cómo tanto para Sexto como para Suárez se configuran nuestras creencias. El desarrollo de esta comparación es precisamente la base que defiende el presente trabajo. $Y$ es que es en base a nuestras creencias que nos preocuparnos por nuestras investigaciones, ya que en ellas surge la cuestión de cuán seguros podemos estar de nuestros juicios o, a lo mejor, cuán falibles son. De esta manera, la creencia es tema de vital importancia para nosotros, pues a partir de los análisis de los pensamientos de Sexto y Suárez deducimos que en cierto sentido las creencias se configuran por nosotros mismos y no nos vienen dadas por alguna objetividad externa que refiera a una naturaleza acabada $y$ absolutamente aprehensible.

A primera vista, parece afirmar Sexto que el escepticismo carece de un sistema, aunque rápidamente precisa en qué sentido es que menciona esto. Sexto nos señala acerca del escepticismo: "También en lo de preguntarnos si el escéptico tiene un sistema nos conducimos de forma parecida. Pues si alguien dice que «un sistema es la inclinación a muchos dogmas que tienen conexión entre sí y con los fenómenos" y llama dogma al asentimiento a una cosa no evidente, entonces diremos que no tiene sistema. / Pero si uno afirma que un sistema es una orientación que obedece a cierto tipo de razonamiento acorde con lo manifiesto, $y$ en el supuesto de que ese razonamiento nos enseñe cómo es posible imaginar correctamente la vida -tomándose el «correctamente» no sólo en cuanto a la virtud, sino en un sentido más amplio- y que se oriente a lo de ser capaces de suspender el juicio: entonces sí decimos que tiene un sistema. / Seguimos en efecto un tipo de razonamiento acorde con lo manifiesto, que nos enseña a vivir según las costumbres patrias, las leyes, las enseñanzas recibidas y los sentimientos naturales" 27 .

Según Sexto el escéptico no tiene un sistema de creencias en cuanto a un sistema fijo e inamovible pues ello resultaría en un sistema dogmático. $Y$ es que para Sexto no se debe asentir a algo que sea no evidente o no manifiesto. Por eso mismo, si se trata por el contrario de razonamientos conforme a lo 
manifiesto y la suspensión del juicio, entonces diremos que el escéptico sí tiene un sistema. Por ello, el escéptico debe razonar conforme a lo manifiesto viviendo acorde a las costumbres, leyes, sentimientos, etc., que aparecen anteél.

Lo que llama la atención del escepticismo pirrónico de Sexto se encuentra en aquella apertura hacia el dinamismo de nuestras creencias. La suspensión del juicio no se restringe a criticar por criticar, sino que básicamente enseña que no debemos fijar creencias conclusivas. No obstante, cabe decir que según Sexto en realidad no es tarea del escéptico el contentarse en las refutaciones por ellas mismas, sino que podemos decir que ellas no deben impedir la búsqueda filosófica ni científica. Podemos agregar de acuerdo a Barnes: "None the less,

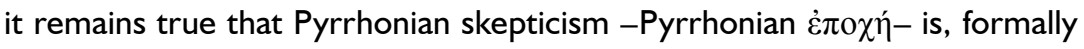
speaking, open-minded and in principle tolerant of future progress" ${ }^{28}$. No se trata, por tanto, del rechazo de las opiniones por el rechazo mismo, sino para no impedir la continuidad de las mismas. Así, es la suspensión del juicio la que precisamente nos permite dinamizar nuestras creencias de este modo.

Por otra parte, es necesario exponer lo que finalmente para Suárez es una naturaleza universal. Y es que Suárez afirma acerca de la naturaleza universal que no es sino dada por las semejanzas y no por alguna unidad real puesta en todas las cosas: "Por esto afirmo, en segundo lugar, que la unidad de la naturaleza universal en cuanto es universal no es real ni se da en las cosas tal como existen en la realidad misma y anteceden a toda operación del entendimiento. (...) Se podrá objetar acaso que en las cosas no se encuentra sólo aquella unidad formal en virtud de la cual cada naturaleza humana, por ejemplo, se dice que es formalmente una en sí misma, sino que se encuentra la unidad en virtud de la cual de todas las naturalezas humanas se dice que tienen la misma razón formal (...), por razón de la que participan de la misma definición, y se dice de todos los hombres que son en realidad de la misma naturaleza. Mas surge una dificultad, porque ésta no es verdadera unidad, sino mera semejanza; en efecto, en la realidad no hay nada verdaderamente uno y realmente indiviso en éste y aquella naturaleza humana, sino que en ésta

28 Barnes, Jonathan, The Toils of Scepticism, Cambridge: Cambridge University Press, 1990, p. II. 
hay sólo algo a lo que a su vez responde algo semejante en la otra naturaleza; esto, empero, no es unidad real, sino semejanza" ${ }^{29}$.

El universal no es una unidad real de la cosa ni tampoco van a anteceder a la operación del entendimiento. Lo que para una cierta tradición la naturaleza formal del ser humano reside en cada uno de ellos por medio de la cual participan en común, para Suárez no está dada sino en cuanto a la semejanza que tienen entre ellos. Por ello, la denominada naturaleza común no es sino la semejanza.

Es aquí donde descansa la postura nominalista de Suárez acerca de los universales, pues el universal no es una unidad real colocada en las cosas, sino que es posterior a la operación del entendimiento: "Por eso de una multitud de cosas en la realidad sólo puede afirmarse que son de la misma naturaleza en el sentido de que son semejantes, pues esta identidad que afirmamos darse entre cosas distintas no puede ser en la realidad más que una semejanza (...)"30. La identidad que encontramos entre varias cosas, por tanto, encuentra su base en las semejanzas que se dan entre las mismas y es forjado por una operación del entendimiento. Añade Heider: "the unity of universal nature qua universal is not real and is not in things, insofar as they exist in reality independently of the operation of understanding" ${ }^{31}$. Debido a que el universal es elaborado por una operación del entendimiento es que no tiene la propiedad de ser algo real fuera de las cosas singulares.

Ahora bien, realizado este examen acerca de las posturas de ambos autores considero que podemos regresar un momento a las palabras iniciales de Sexto acerca del escepticismo pirrónico que propone, pues es aquí donde encontramos la configuración de nuestras creencias que se manifiestan en los juicios a través de los tropos mencionados: "También añadimos lo de «según cualquiera de los tropos» a lo de "en los fenómenos y en las consideraciones teóricas» para que no entremos en cómo se manifiestan los fenómenos o en cómo se forman en la mente las consideraciones teóricas, sino que sencillamente los

29 DM, VI, Sec. II, pp. 719-720.

30 Ibid., p. 720.

3I Heider, Daniel, "Suárez on the Metaphysics and Epistemology of Universals", en: Salas, Victor y Robert Fastiggi (eds.), A Companion to Francisco Suárez, Leiden/Boston: Brill, 2015, pp. 173-174. 
tomemos como aparecen. / Y en absoluto tomamos «proposiciones contrapuestas» como "afirmación y negación»; simplemente como «proposiciones enfrentadas». Y llamamos equivalencia a su igualdad respecto a la credibilidad - no credibilidad, de forma que ninguna de las proposiciones enfrentadas aventaje a ninguna como si fuera más fiable. / La suspensión del juicio es ese equilibrio de la mente por el que ni rechazamos ni ponemos nada. Y la ataraxia es bienestar y serenidad de espíritu”32.

Toda aproximación hacia las cosas según Sexto se da a través de los tropos en los cuales quedó señalado básicamente que las cosas son tomadas como aparecen. $Y$ es que siempre resultan proposiciones contrapuestas acerca de nuestras consideraciones y fenómenos siendo proposiciones equivalentes en cuanto ninguna de las dos tiene porqué ser más fiable que la otra. Así, solo resta la suspensión del juicio para mantener en equilibrio nuestra mente.

Para Sexto la suspensión del juicio es completamente recomendable pues no solo tenemos la capacidad de ejercerla sino que a su vez estamos comprometidos con ella connaturalmente, pues es como una exigencia de nuestra mente el no aceptar dogmáticamente una proposición para que ella llegue a su equilibrio y serenidad. El escéptico de Sexto no consigue la serenidad de espíritu aferrándose a una creencia fija o dogma inamovible, señala Ramos: “(...) sino que ve en ello una afección, un $\pi \dot{\alpha} \theta o \varsigma$, que puede ser curado con la oportuna terapia, con el oportuno abrir los ojos a los múltiples obstáculos que tenemos para conocer la verdad (...)"33. Sabiendo que estamos permanentemente obstaculizados como para entronar creencias fijas, Sexto propone la suspensión del juicio como criterio de investigación.

Por otro lado, Suárez también nos ofrece unas palabras acerca de cómo se elaboran nuestros conocimientos o creencias. Suárez nos muestra una manera de entender la ciencia consecuentemente con la teoría nominalista del conocimiento que suscribe: “(...) la unidad fundamental del concepto objetivo, la cual consiste en la semejanza o conveniencia de muchos singulares en la unidad formal, puesto que basta esto para que el entendimiento, con su propia virtud y eficacia intelectiva, pueda abstraer un concepto objetivo común; de 
donde, para la precisión de dicho objeto común y universal, el entendimiento no supone su objeto, sino que lo elabora, o mejor, le confiere el estado de precisión por denominación extrínseca"34.

Los conceptos objetivos para Suárez que se elaboran a partir de las semejanzas descritas son los que conforman nuestros conocimientos universales. Pero es el entendimiento el que elabora por sí solo dicha naturaleza universal a partir de las semejanzas de las cosas. Así, la elaboración del universal proviene de algo exterior al singular.

El concepto objetivo para Suárez es del que nos valemos para el universal a partir de la elaboración del entendimiento de las semejanzas de las cosas. Para Suárez el universal no es sino obtenido por medio del entendimiento en tanto es elaborado por él, no es obtenido de las cosas singulares como algo que residiera en ellas. Así, podemos decir conforme a Larrainzar: "Será naturaleza universal, por esta razón, la naturaleza descortezada de sus caracteres individuales en intelecciones posteriores a la cognición directa (...)”35. El universal es siempre posterior a los singulares, pero también por esa razón su elaboración no tiene que ver con una unidad real en la cosa singular.

En definitiva, esto guarda una enorme importancia en la comprensión que Suárez maneja acerca de la investigación hacia nuevos conocimientos, pues al ser los universales objeto de nuestras investigaciones no pueden ser pasados por alto en la consideración de una ciencia: "Este es el sentido en que se afirma que la ciencia se ocupa de los universales y no de los singulares, no porque se ocupe de los nombres comunes, sino porque se ocupa de los conceptos objetivos comunes, que aunque no se distinguen de los singulares en la realidad, se distinguen, sin embargo, por razón, siendo esto bastante para todas las expresiones anteriores" 36 .

La ciencia no se ocupa sino de los conceptos objetivos comunes que se distinguen por medio de nuestra razón. Es decir, si bien la ciencia no es un discurso acerca de meros nombres, no hay que olvidar que los conceptos objetivos se

34 DM, VI, Sec. III, pp. 732-733.

35 Larrainzar, Carlos, Una introducción a Francisco Suárez, Pamplona: EUNSA, 1977, p. 117.

36 DM, VI, Sec. VI, p. 750. 
distinguen de los singulares respecto de nuestro entendimiento. La ciencia, por eso, al desarrollarse sobre la base de los conceptos objetivos remite a una revisión de las cosas singulares en su semejanza ${ }^{37}$. Esto es prudente pues de otra manera nuestros conceptos objetivos pueden verse impedidos de brindar un mejor acondicionamiento para desarrollar alguna ciencia.

La configuración de nuestro conocimiento es visto a la luz de esta crítica que podemos hacer hacia él mismo. Y es que para Suárez es importante indicar que no hay que ignorar que en nuestra pretensión de obtener conocimientos no halla limitaciones. Señala Darge: “(...) the objective concept serves as a quasi interface by which one's scientific consideration attains the real thing, and it is the real thing that formally measures the mental activity wherein the theoretical consideration reaches its end"38. Así, nuestra consideración teórica soporta su contenido en relación a lo que ofrece la cosa singular real para la operación de la mente.

\section{$\S 5$. Conclusión}

Para concluir el presente trabajo, podemos afirmar que entre Sexto y Suárez existe un pensamiento similar también sobre este último punto acerca de nuestras creencias. A partir de esta similitud es que podemos realizar una comparación entre el escepticismo pirrónico de Sexto y el nominalismo de Suárez. Donde se sugiere en ambos autores una epistemología de la creencia, pues a pesar de no precisar naturalezas comunes in re o afirmaciones dogmáticas, sí precisan la urgencia de seguir discurriendo acerca de las mismas. Nuestro saber no es sino una elaboración a partir de lo que podemos conjeturar. La ciencia incluso, la cual podríamos pensar muchos que es un discurso objetivo de la realidad, será también considerada como producto de esta

37 Cabe añadir aquí unas palabras de Suárez: "En efecto, nosotros igual que concebimos, así hablamos, de donde resulta que, de la misma manera que nuestros conceptos, aun siendo verdaderos y no falsos, no siempre, sin embargo, son adecuados a las cosas mismas, de igual suerte las palabras se miden por nuestros conceptos, debiendo, por lo mismo, ponernos en guardia para no transferir a las cosas mismas nuestro modo de concebir y para no juzgar a causa de los diversos modos de expresión, que hay verdadera distinción en las cosas, cuando en verdad no la hay" (DM, II, Sec. III, p. 4I0).

38 Darge, Rolf, "Suárez on the Subjects of Metaphysics", en: Salas, Victor y Robert Fastiggi (eds.), A Companion to Francisco Suárez, p. II2. 
configuración de nuestras creencias. Y esto no es otra cosa que la referencia hacia un conocimiento inacabable pero urgentemente requerido de revisión en vista de un mejoramiento continuo.

Es de esta manera que termino el presente trabajo estableciendo el paralelo que buscamos entre las maneras de entender nuestra investigación de cualquier conocimiento tanto para Sexto como para Suárez. Y es que para ambos autores tenemos un pensamiento que busca permanecer fiel primeramente a las sensaciones y cosas singulares. Además de que ambas posturas esbozan la elaboración de nuestros conocimientos a partir de representaciones o conceptos que se construyen siempre en relación a la percepción del individuo y también a las cosas individuales. Finalmente, podemos ver cómo es que tanto para Sexto como para Suárez la configuración de nuestras creencias y nuestra comprensión de lo que es una ciencia descansa sobre la base de lo mencionado anteriormente, en la medida que se pueden imponer ciertas restricciones a nuestra pretensión de verdades conclusivas que escapan incluso a cualquier investigador por el solo hecho de serlo. Tanto para Sexto como para Suárez no deberíamos aferrarnos a pensar que nuestras creencias se correspondan completamente con las cosas y que ello debe remitirnos siempre a una revisión de nuestros juicios acerca de las mismas, ya que somos nosotros mismos los que establecemos todo conocimiento en relación a nuestra concepción. Sin embargo, en ambos autores también vemos una preocupación por ser racional a pesar de no aferrarse a una creencia absolutamente adecuada, lo que sugiere una llamada de atención hacia cualquier dogmatismo especulativo en tanto lo encontraremos asociado al desconocimiento de la real configuración de todos nuestros juicios. En conclusión, doy por demostrado que la suspensión del juicio propio del escepticismo pirrónico de Sexto puede encontrar un paralelo con la teoría nominalista del conocimiento de Suárez. Si consideramos a la noción de creencia en ambos autores desde este dinamismo, podemos decir que Sexto y Suárez son como dos custodios de una misma manera de hacer filosofía. 